\title{
Modelling Steam Generators for Sodium Fast Reactor with Modelica
}

\author{
Franck David Annick Souyri \\ EDF R\&D \\ 6 quai Watier 78400 Chatou - France \\ franck.david@edf.fr annick.souyri@edf.fr \\ Guillaume Marchais \\ Altran Technologie \\ 2 rue P Vaillant Couturier - 92300 Levallois Perret - France \\ guillaume.marchais@altran.com
}

\begin{abstract}
EDF is involved with CEA and AREVA in a common effort for the development of the future nuclear reactor generations. The studies, currently performed by the partners, concentrate on the design of Sodium Fast Reactor types that may include different kinds of innovative circuits and components as compared to the SPX (Super PheniX) plant design.

Based on previous knowledge on SG developed at EDF, with Sodium as hot fluid, and with the help of more recent methods of modeling using the Modelica libraries, a new model for the simulation of steam generator has been developed in order to help the designers of the heat exchangers to meet the requirements for a Sodium Fast Reactor plant design.

The paper will present the current status of the model and a comparison of the results with those of the actual SuperPhenix steam generator database.
\end{abstract}

Keywords: Power plants and energy conversion systems

\section{Introduction}

EDF is involved with CEA and AREVA in a common effort for the development of the future nuclear reactor generations. The studies concentrate on the future design of Sodium Fast Reactor types that may include different kinds of innovative circuits and components as compared to the previous SPX (Super PheniX) design.

Among the numerous subjects of interest driven by the project and technical issues, the present paper will focus on the energy conversion system between the reactor core, where liquid sodium is used as primary coolant, and the steam/water loop.

For safety purpose, i.e. to prevent the consequences of an interaction between water and "primary" sodium in case of tube leaks, an intermediate coupling fluid is required. The resulting design includes two heat exchangers (Figure 1) : the intermediate heat exchanger (IEX) and the steam generator (SG). The SG couples the intermediate fluid with water, which goes through a complete change of phase from liquid water to overheated steam. It is to be noticed that, according to the nominal power of the plant and also for safety reasons, the duty of the heat exchangers (IEX and SG) could be supported by a series of heat exchangers, with the same inlet and outlet conditions of temperature and pressure, rather than by big components.

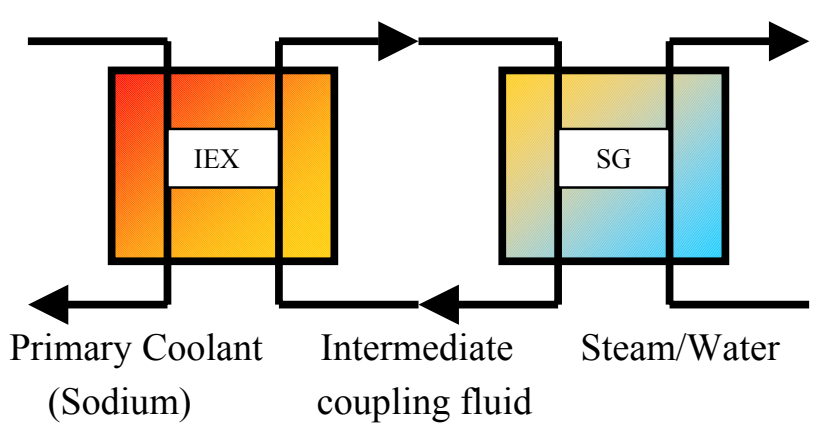

Figure 1 : Global Energy transfer scheme in a Sodium Fast Reactor

Since a final and completed design of the whole system is not decided yet, it was important for $\mathrm{EDF} / \mathrm{R} \& \mathrm{D}$ to develop a model with the capability to integrate any kind of evolutions of geometry or operating conditions and to assess their effects on the energy conversion system. Also, this numerical 
model could offer relevant information for design optimization (especially concerning local temperature behavior on the SG two phase flow side).

The paper presents successively:

- A description of the physical model. This is a 1D model based on equations of conservation for mass, momentum and energy, compliant with transient analysis.

- a comparison of the results of the SG Modelica model with previous studies based on SPX measurements and calculations available at EDF.

- a preliminary assembly of modules to model the global energy conversion system including the connection of two heat exchangers (IEX and SG).

\section{Motivation for the development}

\subsection{EDF's prior experience with Modelica}

In order to improve the efficiency of its simulation tools while reducing their cost, EDF has chosen to use when possible, state-of-the-art readily available tools instead of proprietary codes developed by EDF. The tools used should have open component libraries, be able to perform static and dynamic studies, compute steady states and solve inverse problems. They also should not induce an excessive dependency upon the tool providers. Modelica based tools offer such characteristics. That is why they are considered as good candidates for fulfilling EDF's needs, i.e. modelling and simulation at the system level for the sizing, design verification, validation and operation of its power plants.

In order to evaluate different tools, benchmark cases have been selected, covering the variety of studies made at EDF. Results obtained with our models written with Modelica language are quite satisfactory. Validation studies of the models with Dymola have shown good agreement with previous reference test cases ([1], [2]).

After these first positive results, and also because of the easy use and sharing of the Modelica models with Dymola, EDF decided to develop its own thermofluid Modelica library. The objective is to provide the physical and technological model components needed for steady state and dynamic simulation of power plants under normal and incidental operating conditions. This includes conventional nuclear and thermal power plants, but also future nuclear and thermal plants designs, and systems powered by renewable energy.
The library components must be able to describe single and two-phase flow, with heat transfer when needed, deal with zero and reverse flow, compressible and incompressible flow for water/steam but also other fluids used in specific heat exchangers for example, and smoke networks for thermal power plants.

A Modelica library of $0 \mathrm{D}$ and $1 \mathrm{D}$ thermal hydraulics components has been developed, based on mass, momentum and energy balance equations, completed with closure equations derived from empirical correlations valid for the operating domain under consideration. 1D models describe steam generator and vapour lines. Empirical correlations (heat transfer, pressure losses), adapted to the physical range of operation of the component, have also been translated into Modelica.

The library uses a finite volume approach, based on the staggered grid scheme for space discretization, and the upwind scheme for the handling of flow reversal [3]. Both schemes are well suited for convection, which is the predominant energy transport law within the network. Discretization is performed along the main flow direction only (1D modelling).

The basic model components are divided into two groups: nodes and edges. Nodes represent mixing volumes such as tanks, boilers, splitters and mergers, etc. They implement the mass and energy balance equations. Edges represent flow resistant elements such as valves, simple pressure loss pipes, etc. They implement the momentum balance equations. The network is built by connecting edges to nodes in order to obtain a complete set of mass, energy and momentum equations with their closure equations, and automatically fulfil the numerical scheme requirements. Complex library components such as heat exchangers, evaporator pipes or steam generator can be either developed as independent components or built by assembling edge and node elements.

It is also important to note that EDF has chosen not to use the Modelica inheritance mechanism, in order to keep the readability of the model: the complete set of equations can be found directly in the component model itself, instead of being scattered throughout the library when they are partially derived from super-classes.

\subsection{Specifications for the model}

The purpose of the final model will be to represent two heat exchangers connected with an intermediate fluid. The fluids to be considered are sodium liquid for the hot side at a temperature of about $500{ }^{\circ} \mathrm{C}$ and boiling water for the cold side at a pressure of about 
200 bars. The intermediate fluid could be "classically" sodium liquid as in Phenix or SuperPhenix plants or another fluid to be decided in the future.

The main goal for the model is first to help researchers and pre-designers to assess the performance and the sizes of the different exchangers involved in the process. More over, the requirements for design of pumps will be derived from the calculation of head losses through the different parts of the components.

A second objective assigned to the model is to provide accurate local parameters such as velocity, temperature, pressure, void fraction,... in order to check if the parameters comply well with other concerns like fluid-structure interaction, corrosion and so on.

In a final step, the model will have to calculate off design situations or transients with respect to actual physical phenomenon and could be used in connection with the entire energy conversion system.

\section{Model description}

\subsection{Two generic modules}

To meet the above requirements, it was decided to develop separate modules for the fluids and for tube wall between the hot side and the cold side.

For our applications, the heat exchangers components can be considered as one dimensional with counter-flows even though the local flow can be locally perpendicular to the tube arrays.

Concerning the steam/water size, the fluid is supposed to enter at liquid sub-cooled conditions, then is heated and goes through a complete change of phase to become steam at super-heated conditions at the outlet. During the process, due to the phase change, the physical mechanisms that drive the heat transfer and pressure are very complex and differ strongly between the inlet and outlet of the tubes.

The complex mechanisms are represented by empirical correlations depending on geometry, direction of the fluid and local parameters (velocities, pressure, quality..). It appears clearly that it is necessary to have a local description of the average physical parameters all along the tubes to represent the actual physics.

As a result, hot side, tube wall and cold side modules are connected together, and represent a "cell" with a limited length (figure 2). The cells can then be connected together to form the whole length of the global heat exchanger.

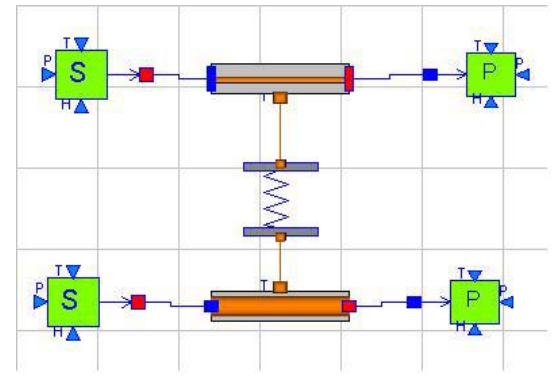

Figure 2 : generic cell with two modules for fluids and one module for the tube

The physical models employed should be able to calculate one phase or two-phase flows within the fluid module and heat transfers through the tube wall.

For a fluid module, the 1D model is based on a classic set of equations for the conservation of mass, momentum and energy (as given in equations 1,2 and 3).

$$
\frac{\partial \rho_{m}}{\partial t}+\frac{\partial}{\partial l}\left(\rho_{m} \cdot V_{m}\right)=0
$$

$$
\begin{array}{r}
\frac{\partial \rho_{m} V_{m}}{\partial t}+\frac{\partial}{\partial l}\left[\rho_{m} V_{m}^{2}+\alpha(1-\alpha) \frac{\rho_{l} \rho_{v}}{\rho_{m}} V_{v l}^{2}\right] \\
+\frac{\partial P}{\partial l}-F_{m}-\rho_{m} \cdot g=0
\end{array}
$$

$$
\begin{aligned}
& \frac{\partial \rho_{m} H_{m}}{\partial t}+\frac{\partial}{\partial l}\left[\rho_{m} V_{m} H_{m}+\alpha(1-\alpha) \frac{\rho_{l} \rho_{v}}{\rho_{m}} L V_{v l}\right] \\
& -\frac{\partial P}{\partial t}-\frac{\partial P}{\partial l}\left[V_{m}+\alpha(1-\alpha) \frac{\rho_{l}-\rho_{v}}{\rho_{m}} V_{v l}\right]-\Phi_{m}=0
\end{aligned}
$$

where :

$-\rho \mathrm{V}, \mathrm{H}$ and $\mathrm{P}$ are respectively the density, velocity, enthalpy and pressure of the fluid. The fluid is considered as an homogenous mixture (subscript $\mathrm{m}$ ) in case of two-phase flow conditions.

- $\alpha$ is the void fraction (ratio of volume occupied by steam on total volume of fluid). Moreover, we have the relation: $\rho_{m}=\alpha \rho_{v}+(1-\alpha) \rho_{l}$

where $\mathrm{v}$ and 1 indicate respectively the vapor and liquid phase

- L is the difference of enthalpy between vapor and liquid at saturation condition for a given pressure 
- $\mathrm{V}_{\mathrm{vl}}$ is the difference of velocity between the steam and the liquid that results from different forces acting on each individual phase

- $F_{m}$ is set for the sum of forces (friction, viscosity, obstacles..) acting on the mixture; $g$ is the constant of gravity.

- $\Phi_{\mathrm{m}}$ represents the heat transfers between the fluid and the tube walls. It can be expressed by the following equation :

$\Phi_{m}=\frac{4}{d_{t h}}\left[h_{t m}\left(T_{t}-T_{m}\right)\right]$

where $T_{t}$ and $T_{m}$ are respectively the temperatures of the tube and the fluid; $\mathrm{d}_{\mathrm{th}}$ is the thermal diameter and $\mathrm{h}_{\mathrm{tm}}$ is the heat exchange coefficient between tube the tube and the fluid.

The 3 main unknowns solved by the solver from this 3 equations are, after a combination of equations, the pressure $\mathrm{P}$, the dynamic enthalpy $\mathrm{H}_{\mathrm{m}}$ and the mass flow rate $\mathrm{Q}_{\mathrm{m}}=\rho_{\mathrm{m}} \mathrm{V}_{\mathrm{m}}$.

To characterize the two-phase mixture, it is assumed that the phases are always at thermodynamic equilibrium and that the relative velocity $\mathrm{V}_{\mathrm{vl}}$ is known thanks to a relevant empirical correlation.

The terms $F_{m}$ is given by an empirical correlations depending on geometry and local fluid parameters.

The tube module includes a unique equation of energy balance in the tube wall (see equation (6)). The equation links the time dependent variation of the tube wall temperature with the surface heat fluxes on both sides of the tube.

$\rho_{t} \cdot C p_{t} \cdot \frac{\partial T_{t}}{\partial t}=-\Phi_{1}-\Phi_{2}$

where $\mathrm{Cp}_{\mathrm{t}}$ is the thermal capacity of the tube and $\Phi_{1}$ and $\Phi_{2}$ the surface heat fluxes which can be written as equation (5).

It is necessary to take account of the thermal conductivity of the tube. This is made by means of the following relation (to be used for in-tube side):

$h_{t m}=\frac{h_{t w}}{1+\mathrm{A} \cdot h_{t w}}$ with $\mathrm{A}=\frac{D_{i}}{2 \cdot \lambda_{t}} \cdot \operatorname{Ln}\left(\frac{D_{i}+e}{D_{i}}\right)$.

where $D_{i}$ is the internal diameter of the tube and e its thickness; $h_{t w}$ is the heat exchange coefficient on tube wall and $\lambda_{t}$ the conductivity. A quite similar formula adapted to outer tube sides is also required.

Finally, the thermal properties of the fluids and materials are given either by the thermodynamic tables in the standard version of Modelica or by specific models.
This set of equations, with the complementary assumptions, was successfully used for SG modeling with Modelica in PWR applications [1]. Furthermore, the model is also widely employed at EDF in a 3D formulation to calculate two-phase flows in SG or reactor cores in PWR plants with a CFD component code [4].

\subsection{Closure laws}

All the correlations used in our model to calculate friction factors or heat exchange coefficients are taken from relatively old EDF's references [5]. Nevertheless the set of correlations used was adapted to represent correctly the physics in a SG heated by sodium liquid.

In this particular design, the exchanger consisted of helically coiled tubes and the correlations take into account these unusual features. The one phase sodium flows quite perpendicularly across the tube bundle. One phase or two-phase steam water flows inside tubes that are curved.

Since we didn't find any indication about relative velocities between the phases, the $\mathrm{V}_{\mathrm{vl}}$ term is set to 0 .

\subsection{Input/output of the modules}

The connections between the modules are as follow:

- Inlet connector of fluid modules consists of enthalpy and mass flow rate; outlet connector includes the pressure variable.

- The connection of tube wall module with fluid module exchanges the average tube temperature and the surface heat fluxes.

\section{Application to a Steam Generator of Super Phenix}

The objective of the work presented below was to validate the capability for the model to represent correctly the behavior of an helically coiled steam generator heated with sodium.

To be more precise, one must check the correct implementation of the modules, the reliability of correlations by comparing the calculations with test results or calculations made with an older code that is no longer available.

The database for the comparisons comes directly from the test campaign performed on the SG " $D$ " of SPX plant at different power levels. Moreover, we used the results of an old code to compare temperature and pressure profiles for one phase sodium flow and two-phase steam water flow. 


\subsection{Geometrical features and operating condi- tions}

A general view of the SG design is given in figure 3 . The SPX Steam Generator was an helically coiled tubes heat exchanger. The sodium was flowing downwards on the shell side whereas the water was flowing upwards and was boiling inside the tubes of Incoloy 800.

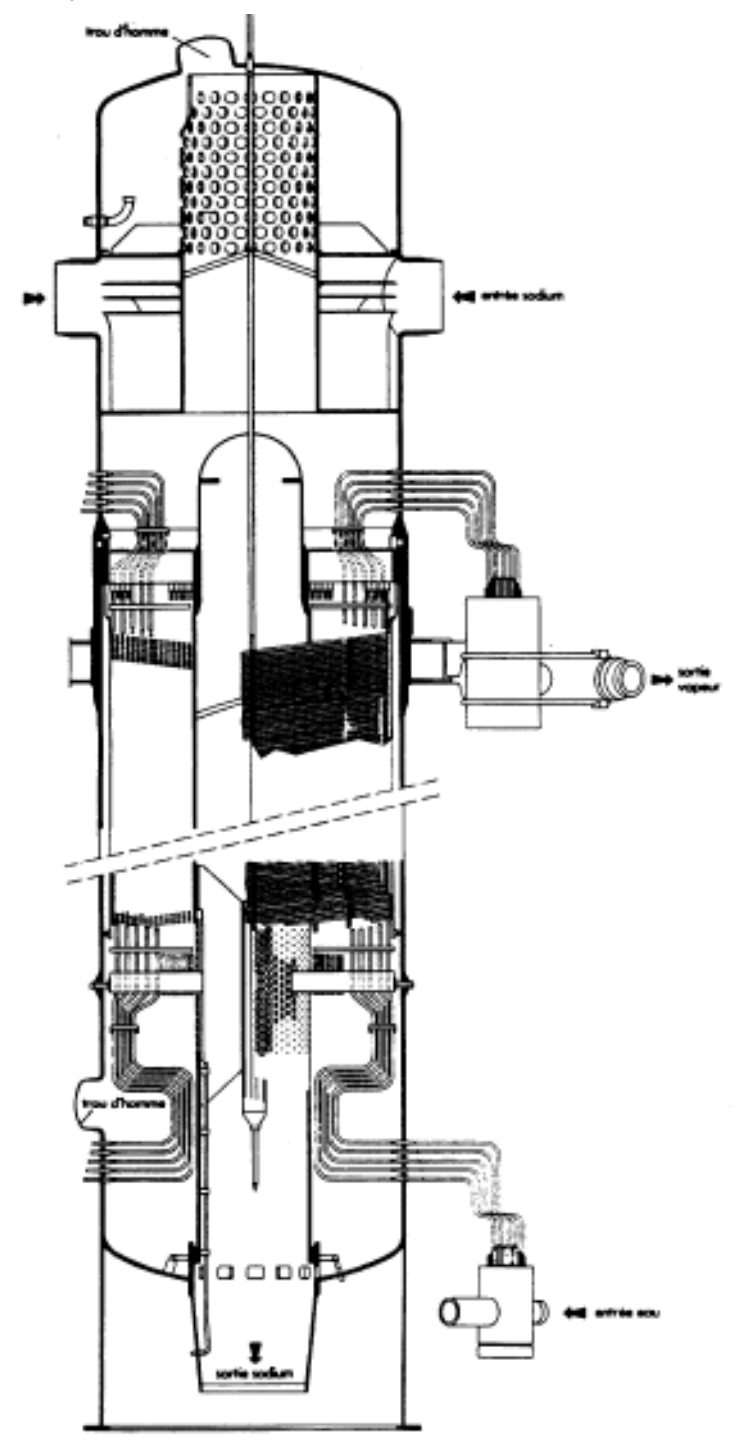

Figure 3 : Plan of SPX Steam Generator

Table 1 : Tube bundle data

\begin{tabular}{lr}
\hline Inner diameter & $19,8 \mathrm{~mm}$ \\
Outer diameter & $25,0 \mathrm{~mm}$ \\
Coil $^{\text {st }}$ diameter & $1,17 \mathrm{~m}$ \\
Coil pitch & $45 \mathrm{~mm}$ \\
Coil number & 17 \\
Tube number & 357 \\
Helical angle & $7,45^{\circ}$ \\
\hline
\end{tabular}

The bundle was contained in an annulus of inner diameter $1,125 \mathrm{~m}$ and external diameter 2,655 $\mathrm{m}$.

Main characteristics and layout of the bundles are presented Table1. The tube bundle is completed with straight and curved parts to be connected to water supply and steam outlet pipes.

The heated part covers a height of about $14,9 \mathrm{~m}$ and corresponds to an average tube length of $90,9 \mathrm{~m}$.

Operating conditions at nominal power during the tests are presented Table 2 .

Table 2 : Operating conditions (measured) near $100 \%$ of nominal power

\begin{tabular}{lr}
\hline Power exchanged & $724 \mathrm{MW}$ \\
Sodium inlet temperature & $518^{\circ} \mathrm{C}$ \\
Sodium outlet temperature & $343^{\circ} \mathrm{C}$ \\
Sodium mass flow rate & $3249 \mathrm{~kg} / \mathrm{s}$ \\
Water inlet temperature & $233,8^{\circ} \mathrm{C}$ \\
Water outlet temperature & $489,8^{\circ} \mathrm{C}$ \\
Water mass flow rate & $331 \mathrm{~kg} / \mathrm{s}$ \\
Steam outlet pressure & $185,4 \mathrm{bar}$ \\
\hline
\end{tabular}

\subsection{Calculation results and comparison with tests}

Beyond the instrumentation dedicated to global performances and inlet and oultet flow parameters, local instrumentation (60 thermocouples) was set at different levels in the sodium side and at the outlet of steam water side. The measurements were performed by Novatome during 1986.

The two categories of measurements provide global and local information and are used in the following chapter for comparison with calculation results. Nevertheless, local steam temperature measurements were not documented in EDF's reports and can not be used.

However, global calculations and profiles are compared with EDF's older code that was validated with the present data and with a $45 \mathrm{MW}$ experiment [6] having similar geometrical features.

For our simulations, a number of 20 "cells" was used to represent the whole length of the tubes. That number is a balance between the complexity of the model and its capability to "catch" the changes of phase and local physical behavior.

Moreover, a specific arrangement was used to take into account a by pass of sodium at the bottom of the bundle (see detail Figure 4). 
For the two fluids, input data are temperature and mass flow rate at the inlet and pressure at the outlet.

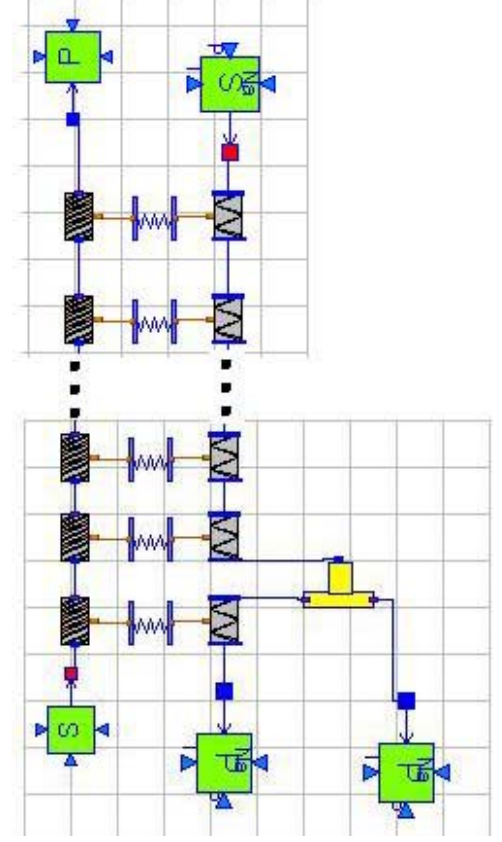

Figure 4 : sodium bypass arrangement

\section{Calculation results at nominal power:}

The first comparison was made for a thermal power exchanged $(724 \mathrm{MW})$ very close to the designed nominal power $(750 \mathrm{MW})$. It was necessary to slightly adjust the calculated thermal power that was $2 \%$ under the expected one. This was achieved by tuning the conductivity of tube material.

Figures 5 and 6 present respectively the profiles of temperatures and steam water pressure calculated along the tubes with EDF's previous code. The dots that appear in Figure 5 come from temperature thermocouples located at different levels in the shell side (i.e. where the sodium liquid flows). The comparison was satisfactory, so we can rely on calculations with the previous code.

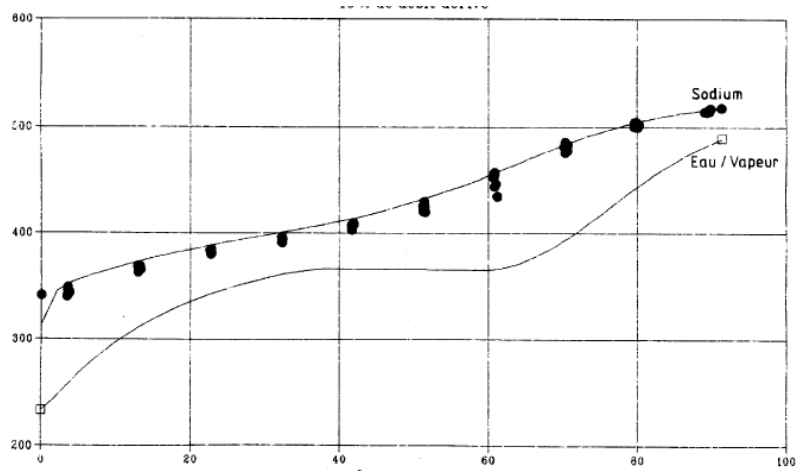

Figure 5 : temperature profiles (upper curve is sodium, lower curve is steam water) - calculated with previous code
The steam/water temperature profile shows the different parts where the fluid is liquid, two-phase or vapor. The minimum of temperature pinch appears at the upper part of the tubes but near the beginning of boiling section the temperature pinch is also narrow.

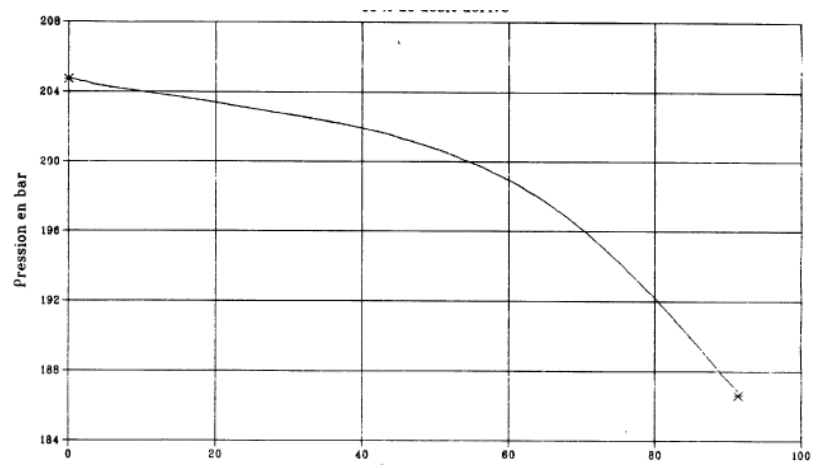

Figure 6 : Pressure profile on steam/water side, calculated with previous code

Unsurprisingly, the pressure profile on Figure 6 shows different slopes depending on whether one area is liquid (left hand side), two-phase (center) or vapor (right hand side).

Figures 7 and 8 present the calculation results of temperatures and steam/water pressure profiles with our Modelica current modeling.

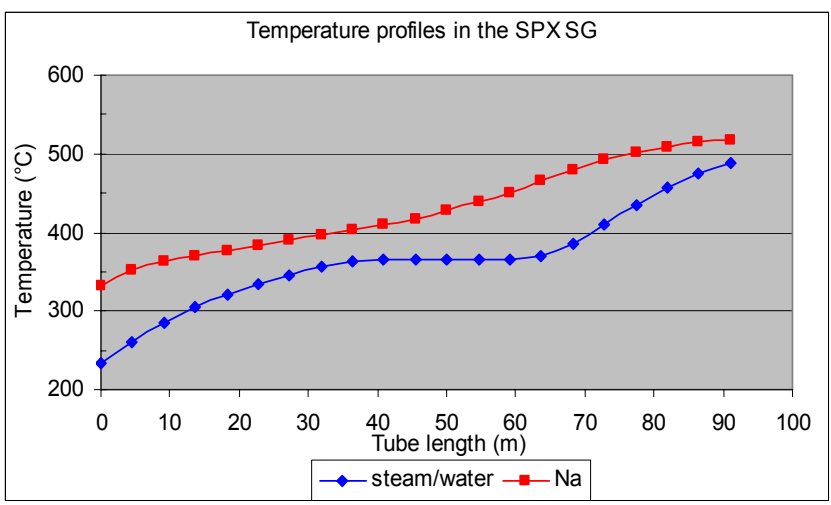

Figure 7 : temperature profiles along the tubes with Modela model

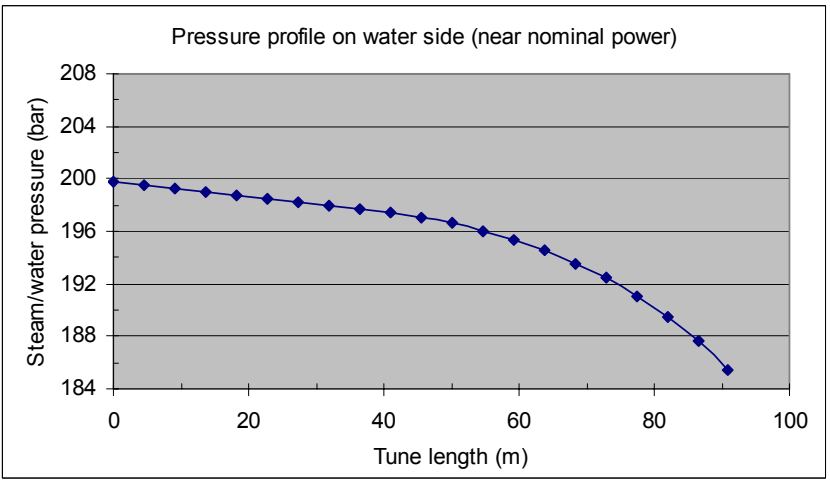

Figure 8 : pressure profile along the tubes with Modela model 
As it can be seen on figures 5 and 7, the temperature profiles compares perfectly. For the two simulations, beginning and end of boiling sections are located at the same tube heights.

Although the shapes of pressure profiles (figure 6 and 8) are quite similar, there is a significant difference between the values of pressure drops of about 2,5 bar. The difference is concentrated in the upper part of the tubes at high two-phase flow quality and beyond. That may be due to the relative velocity set to 0 in our model or friction factors in two-phase flow.

This difference will have to be investigated in a future work.

Results at intermediate power exchanged (50\% and 25\% of nominal power):

Two other calculations were made with the same physical assumptions and closure relation ships for two thermal powers of 388 and $151 \mathrm{MW}$.

The calculations compare very well with the previous ones : differences of power exchanged remain below $1,5 \%$.

\section{Toward the modeling of a complete EI/SG system}

The calculation results presented in chapter 4 are very satisfying. It is now possible to move with confidence to a more complete modeling of the coupled system with two heat exchangers (EI and SG) as discussed in chapter 1.

Main objective for this work is first to bring out a first geometrical design that complies with plant requirements. More over, it is suggested to study other fluids than sodium as coupling fluids.

Figure 9 presents a possible and very preliminary architecture that couples an Intermediate Heat Exchanger with a Steam Generator. The intermediate fluid can be chosen by entering the right thermophysical properties in the modules.

To perform this work, all useful parameters for the geometrical design are lumped in a specific "Geometry" module. Each generic module will find current geometrical parameters values in the "Geometry" module. Moreover, the "Geometry" module contains all the specific and coherent calculations between the geometry features and it is used to help the designer to find out geometrical parameters if another one is specified.

Once all the geometric details have been set for the model, the calculation allows to check the technical constraints (total power exchanged, pressure drops, local parameters such as temperatures or velocities..).

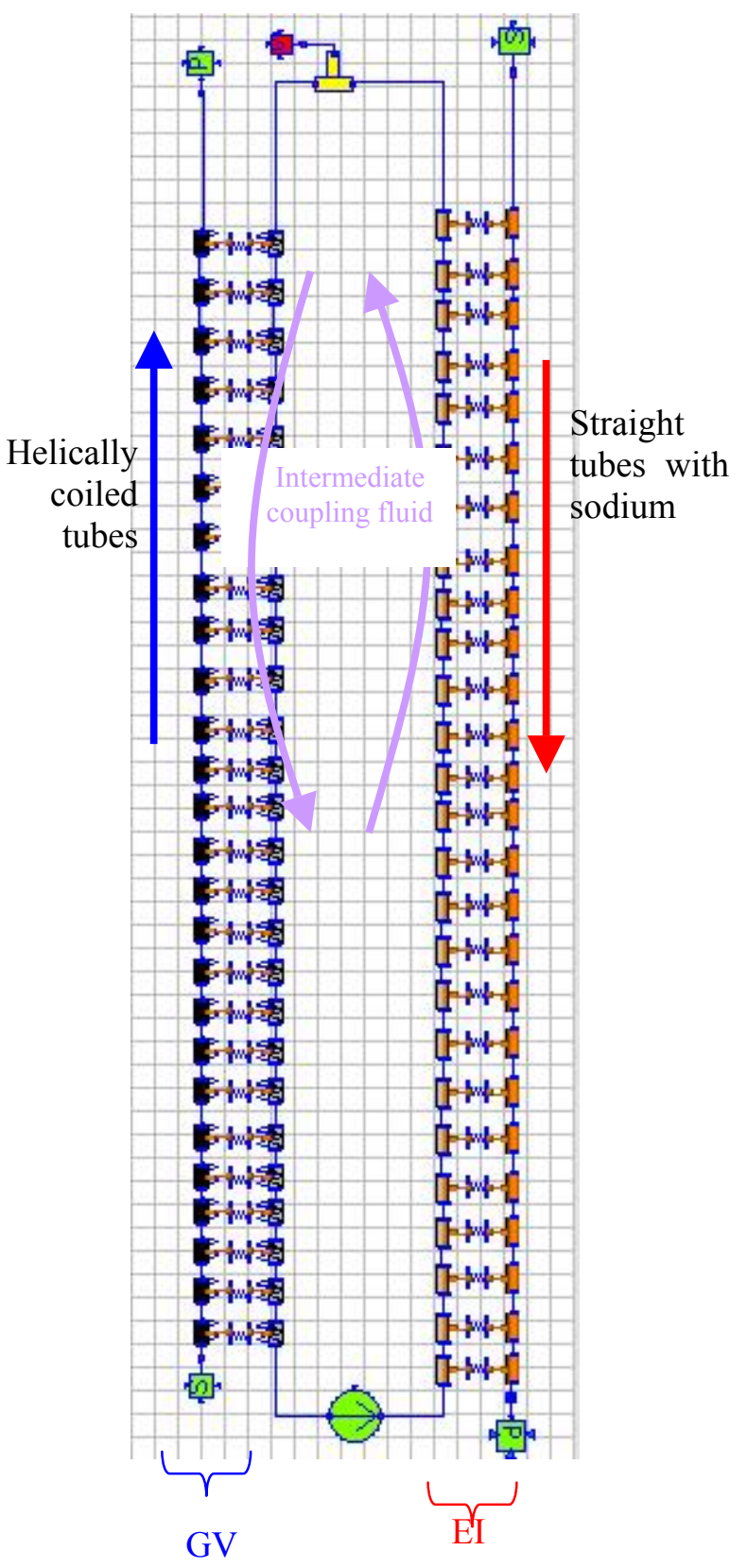

Figure 9 : architecture of coupled heat exchangers

\section{Conclusions}

In the framework of the studies for development of future Sodium Fast Reactor, the Modelica library is used, at EDF/R\&D, to model the systems of heat exchangers coupling the primary loop with sodium to the steam/water flows by means of an intermediate coupling fluid.

The work presented here is focused on the development of a physical model to represent a Steam Gen- 
erator made of helically coiled tubes and heated with liquid sodium.

The set of equations, the closure laws and their correlations form a comprehensive model that shows its capability to calculate correctly the global heat exchanges and the temperatures and pressure profiles along the tubes, as compared to SuperPhenix Steam Generator database and previous modeling.

On this basis, the model will be used in near future to participate in the design of the complete and coupled system including an Intermediate Heat Exchanger and a Steam Generator.

\section{References}

$[1]$

Avenas C. et al, "Quasi-2D Steam Generator Modelling with Modelica”, ISC'2004, Malaga, Spain.

[2]

Souyri A. et al., "Pressurized Water Reactor Modelling with Modelica", $6^{\text {th }}$ international Modelica Conference, March 3-4, 2008, Bielefeld, Germany

[3] Patankar S.V., "Numerical Heat Transfer and Fluid Flow", Hemisphere Publishing Corporation, 1980.

[4] David F., "Three dimensional thermal-hydraulic simulation in steam generators with THYC Exchanger code - Application to the UTSG model 73/19", NURETH9, October 3-8, San Francisco, Californie

[5] Fontaine et al., "Synthèse d'essais de maquettes d'un générateur de vapeur chauffé au sodium", Nuclear energy maturity, Paris, 1975,716-737

[6] Lecoeuvre JM et al., "Numerical simulation of operation of the model for the Super-Phenix steam generator in non-stationary regime", EDF, Bulletin de la Dierction des Etudes et Recherches, Serie A Nucleaire, Hydraulique, Thermique $\mathrm{N}^{\circ} 3$, 1981, pp 27-33 